\title{
Delirium, Frailty, and Fast-Track Surgery in Oncogeriatrics: Is There a Link?
}

\author{
Fiammetta Monacellia, d Alessio Signori ${ }^{b}$ Matteo Prefumo a, d \\ Chiara Giannotti ${ }^{a}{ }^{d} \quad$ Alessio Nencioni ${ }^{a}{ }^{d} \quad$ Emanuele Romairone ${ }^{c}$ \\ Stefano Scabini ${ }^{c}$ Patrizio Odettia

\begin{abstract}
${ }^{a}$ Hospital Policlinic San Martino, Section of Geriatric Medicine, Department of Internal Medicine and Medical Specialties (DIMI), University of Genoa, Genoa, Italy; ${ }^{b}$ DISSAL,

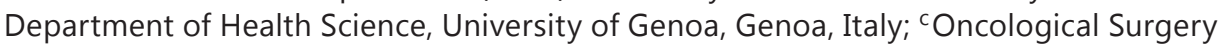
and Implantable Systems, Hospital Policlinic San Martino, Genoa, Italy; ${ }^{\text {dI.R.C.C.S. per }}$ I'Oncologia, Hospital Policlinic San Martino, Genoa, Italy
\end{abstract}

\author{
Keywords \\ Delirium · Functional impairment - Geriatrics · Neuroinflammatory aspects of dementia and \\ delirium
}

\begin{abstract}
Background/Aims: Postoperative delirium (POD) is more frequent in elderly patients undergoing major cancer surgery. The interplay between individual clinical vulnerability and a series of perioperative factors seems to play a relevant role. Surgery is the first-line treatment option for cancer, and fast-track surgery (FTS) has been documented to decrease postoperative complications. The study sought to assess, after comprehensive geriatric assessment (CGA) and frailty stratification (Rockwood 40 items index), which perioperative parameters were predictive of POD development in elderly patients undergoing FTS for colorectal cancer. Methods: A total of 107 consecutive subjects admitted for elective colorectal FTS were enrolled. All patients underwent CGA, frailly stratification, Timed up \& go (TUG) test, 4AT test for delirium screening, anesthesiologists physical status classification, and Dindo-Clavien classification. Results: The incidence of POD was $12.3 \%$. Patients' prevalent clinical phenotype was pre-frail. The multivariate analysis indicated physical performance (TUG in seconds) as the most significant predictor of POD for each second of increase. Conclusions: Only few procedure-specific studies have examined the impact of FTS for colorectal cancer on POD. This is the first study to investigate the risk factors for POD, in a vulnerable octogenarian oncogeriatric population submitted to FTS surgery and frailty stratification.


Monacelli et al.: Delirium, Frailty, and Fast-Track Surgery in Oncogeriatrics

\section{Introduction}

Postoperative delirium is a common clinical condition in elderly patients undergoing surgery, ranging from 28 to $50 \%[1,2]$. The interplay between patient's clinical vulnerability and a series of perioperative variables is considered a major determinant [3-6]. Due to the aging population, colorectal cancer has been continuously increasing, and surgery is the firstline effective treatment option, shifting to less invasive interventions because of better postoperative technical results [7].

The concept of fast-track surgery (FTS) has been developed and documented to be successful by decreasing postoperative complication rate, length of stay, comorbidity, and convalescence [8]. This new model of care is based on a combination of unimodal evidencebased care interventions; it optimizes nutrition, decreased use of tubes, drains, and catheters, mechanical bowel preparations, early mobilization, and multimodal nonopioid analgesia, compared to traditional surgery.

Most of the evidence on postoperative delirium (POD) derives from cardiac and orthogeriatric settings; so far, its association with oncogeriatrics and, in particular, solid cancer resections has received less attention [9-11]. Recently, it has been found that colon rectal surgery was associated with higher postoperative delirium and poorer clinical outcomes, including length of hospital stay and mortality [12]. Similarly, age, past history of delirium, and the operative approach were risk factors for POD after colorectal cancer surgery [7]. In particular, the laparoscopic procedure was significantly associated with lower POD incidence [7]. Remarkably, a cornerstone randomized clinical trial [13] compared FTS with traditional perioperative protocols and their impact on a set of postoperative complications in oncogeriatric patients undergoing colorectal surgery. The major findings indicated that FTS reduced the length of stay and postoperative complications, including delirium. So far, opportunities for earlier interventions in patients with cancer who are increasingly susceptible to delirium by virtue of surgical elective interventions are warranted.

The objective of this study was to investigate which preoperative, intraoperative, or postoperative parameters were predictive of POD development in elderly patients undergoing elective FTS for colorectal cancer and frailty assessment.

\section{Material and Methods}

This was a cross-sectional study performed in the oncological gastrointestinal surgery ward of Ospedale Policlinico San Martino, Genoa, Italy.

\section{Patient Selection}

Between January and December 2016, 107 consecutive patients admitted for elective colorectal FTS [8] were enrolled after obtaining their written informed consent. The study was approved by the Local Ethical Committee and met the guidelines of the local Governmental Agency.

Patients were included if they were $>70$ years old, had a first diagnosis of colorectal cancer (according to the 5th edition of the TNM staging system) and were scheduled for elective FTS according to the FTS protocol illustrated in Table 1.

Patients were excluded if they were younger than 70 years, had a previous history of delirium, had a scheduled intervention for cancer relapse or palliative intervention, had previous neo-adjuvant radiotherapy or chemotherapy treatment, or had any clinical instability needing acute surgery. 
Table 1. Fast-track colorectal surgery protocol

Preoperative assessment

Anesthesiology assessment

Comprehensive geriatric assessment

Cardiologist visit if needed

Diabetologist visit if needed

Pulmonologist visit if needed

Nutritionist visit for tailored nutritional intervention along with standard oral hypercaloric supplementation (2 days, $600 \mathrm{kcal} /$ day) if needed

Physiotherapist assessment for pre-and postoperative rehabilitative plan and training if needed

Intraoperative assessment

Peridural catheter or peripheral venous access for pain control

General anesthesia with propofol and remifentanil by target control technique

Total intravenous liquid infusion (saline solution $0.9 \% 6 \mathrm{~mL} / \mathrm{kg} / \mathrm{h}+500 \mathrm{~mL}$ hydroxyethylamide 130/0.4)

Mechanical or physical devices to maintain normothermia if needed

Ondansetron $4 \mathrm{mg} 30 \mathrm{~min}$ before intubation

Hemotransfusion if blood pressure $<20 \%$ estimated basal value

Droperidol $0.625 \mathrm{mg}$ after surgery

Urinary catheter placement/drainage placement/peripheral venous access placement

Nasogastric tube placement and removal after surgery

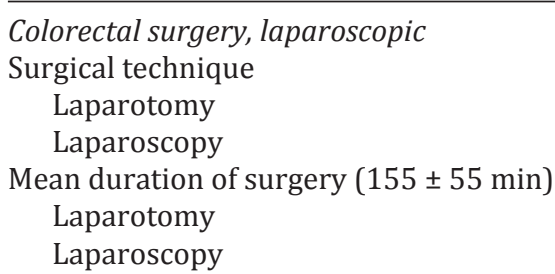

Postoperative assessment - first day

Analgesic control: Peridural catheter or by peripheral venous access with acetaminophen 1 g i.v. $\times 4 /$ day and tramadol $100 \mathrm{mg}$ i.v. if needed (pain control: numeric rating scale [NRS] <4/10)

Trunk control and patient seated for at least $2 \mathrm{~h}$ a day

Respiratory rehabilitation for 10 min a day

Early oral liquid assumption (maximum $1 \mathrm{~L}$ a day) if possible

Oral nutritional supplementation (protein and caloric supplementation $300 \mathrm{kcal} /$ day per single supplement) if possible or parenteral i.v. nutrition $(1,000 \mathrm{~mL} /$ day, $700 \mathrm{kcal} /$ day $)$ for 5 consecutive days after surgery

Bowel evacuation daily and peristalsis assessment

Postoperative assessment - day 2 to day 5

Analgesic control: acetaminophen $1 \mathrm{~g} \times 4$ /day and tramadol $100 \mathrm{mg} /$ day if needed (pain control: NRS <4/10)

From day 3 to day 5 after surgery: pain control: peridural catheter withdrawal and oral analgesic therapy: paracetamol $300 \mathrm{mg}$ and oxycodone $5 \mathrm{mg} 3$ tablets a day OR paracetamol $300 \mathrm{mg}$ and oxycodone $10 \mathrm{mg}$ 3 tablets a day (pain control: NRS <4/10); if needed, ketorolac $30 \mathrm{mg}$ i.m. (maximum $90 \mathrm{mg}$ in $24 \mathrm{~h}$ )

Trunk control and patient seated for 6-8 h a day

Walking rehabilitation from 3 to 5 times a day

Respiratory rehabilitation for 10 min 4 times a day

Early oral liquid assumption (maximum $1 \mathrm{~L}$ a day) if possible

Oral nutritional supplementation (protein and caloric supplementation) if possible or parenteral i.v. nutrition (1,000 mL/day, $700 \mathrm{kcal} /$ day) for 5 consecutive days after surgery

Bowel evacuation daily, peristalsis assessment, and normal bowel movement

Parenteral nutrition withdrawal and oral normal feeding (3 meals a day) with oral nutritional supplementation ( 2 a day, if needed)

Withdrawal surgical drainage

Withdrawal urinary catheter 
Table 2. Factors precipitating POD in elective fast-track surgery oncogeriatric patients: standardized clinical parameters according to fast-track protocol

Nutrition

Standardized clinical protocol for nutrition: oral supplementation (300 kcal/day for 1 supplement day and/or i.v. parenteral nutrition (1,000 mL i.v./day for 5 days; $700 \mathrm{kcal} /$ day)

Devices

Standardized device use (peripheral venous access, urinary catheter, and abdominal drainage)

Bowel movement

Standardized clinical protocol for constipation (osmotic agents for 7 days) with daily report of patient

bowel evacuation and movement

Pain

Standardized clinical protocol for pain (i.v. paracetamol $1 \mathrm{~g}$ a day for $48 \mathrm{~h}$ ) and additional narcotics given above standards record

Physical constraints

Standardized application of bedrail constraints for $72 \mathrm{~h}$

Plasmatic determination of hemoglobin, creatinine, sodium, potassium, C-reactive protein, after 48/72 h

from surgery

Incident postoperative drug administration record

Postoperative blood transfusion record

Factors Predisposing to Delirium, Comprehensive Geriatric Assessment and Frailty Status

Patients' clinical characteristics were assessed at hospital admission and included sociodemographic and comprehensive geriatric assessment (CGA) [14]. The latter included: cognitive status (Mini-Mental State Examination [MMSE] and Shulman I Clock Drawing Test) $[15,16]$; functional status (Barthel Index and Instrumental Activities of Daily Living) $[17,18]$; comorbidity (Cumulative Illness Rate Scale for Geriatrics) [19]; depression (Geriatric Depression Scale) [20]; malnutrition (Mini-Nutritional Assessment) [21]; risk of falls (Tinetti Scale) [22]; and pain (Numeric Rate Scale). A CGA score of $>3$ defined patients as frail and a score of $2<\mathrm{CGA}>3$ defined patients as pre-frail.

All patients underwent frailty index assessment based on the Rockwood 40-item index [23]: a score of $\leq 0.09$ defined patients as fit; a total score of $\geq 0.25$ as frail and a score between 0.08 and 0.25 as pre-frail. All patients underwent ECOG Performance Status (ECOG PS) [24] oncological assessment, anesthesiologists physical status [25], and the Timed up \& go test (TUG) [26] to assess physical performance.

Delirium was diagnosed by an experienced geriatrician using DSM-V criteria at baseline [27]. This same geriatrician was in charge of patients postoperatively as part of a multidisciplinary assessment of elderly surgical patients in our hospital.

Moreover, delirium was also assessed by a second independent geriatrician, using a rapid assessment test for delirium (4AT) [28] after $48 \mathrm{~h}$ from surgery. 4AT is a recently developed and validated screening tool for the assessment of delirium in geriatric patients. Patients who scored $\geq 4 / 12$ on the 4 AT test were also assessed with the Delirium Motor Subtype scale [29] for the evaluation of delirium psychomotor subtype.

The postoperative complications rate was recorded according to Dindo-Clavien classification [30] along with non-surgical-related adverse events. Postoperative blood transfusions were also registered. The perioperative mortality (after 7 days) and 1 month mortality rates were calculated regardless of whether the death occurred in hospital or after discharge. The number of drugs taken by the patients was also collected. 
Table 3. Patients' clinical characteristics based on Comprehensive Geriatric Assessment (CGA) and Rockwood Frailty Index (40-item FI)

\begin{tabular}{lr}
\hline Assessment tool & Mean $\pm \mathrm{SD}^{\mathrm{a}}$ \\
\hline MMSE & $27.13 \pm 0.35$ \\
CIRS & $4.39 \pm 0.19$ \\
CDT & $2.57 \pm 0.15$ \\
4AT test & $3.47 \pm 0.23$ \\
Tinetti & $24.29 \pm 0.57$ \\
MNA & $23.32 \pm 0.32$ \\
Barthel index & $97.68 \pm 0.65$ \\
IADL & $7.19 \pm 0.16$ \\
GDS & $3.62 \pm 0.32$ \\
Gijon scale & $8.71 \pm 0.26$ \\
NRS & $0.60 \pm 0.18$ \\
TUG & $10.84 \pm 0.59$ \\
CGA & $3.48 \pm 0.23$ \\
Rockwood FI (40 items) & $0.23 \pm 0.01$ \\
ASA & $2.28 \pm 0.07$ \\
Dindo-Clavien & $1.07 \pm 0.12$ \\
SF36 & $0.73 \pm 0.05$ \\
Karnofsky & $89.38 \pm 1.19$ \\
ECOG PS & $0.37 \pm 0.06$ \\
Mean drugs & $4.82 \pm 0.29$
\end{tabular}

SD, standard deviation. For other abbreviations, see Table 4 a 97 patients: no missing data.

Factors Precipitating Delirium: Postoperative Clinical Assessment

The optimization of factors precipitating POD, on the basis of the FTS protocol, is illustrated in Table 2.

\section{Statistics}

Results were reported as mean \pm standard deviation. Factors predisposing to and precipitating delirium (POD) were analyzed prospectively, comparing the delirious group with the nondelirious group of patients, based on the $4 \mathrm{AT}$ score (cut-off $\geq 4$ ). The parametric T test was used to compare delirious and nondelirious patients on quantitative measures. All significant measures at univariate analysis were selected with a stepwise approach to be included into the multivariable model, adjusted for age and gender. A $p$ value of 0.10 was used as threshold for inclusion into the model and $p<0.05$ was considered statistically significant. Graph Pad v.5.0b and Stata (v.14; StataCorp) were used for the computation.

\section{Results}

Patients' clinical characteristics are illustrated in Table 3. The mean age was $80.26 \pm 0.65$ years (female 73 and male 34 years). Patients had surgery for colon carcinoma in $71 \%$ ( $n=$ 72 ) of cases and rectum carcinoma in $29 \%(n=35)$ of cases.

Seventy percent $(n=77)$ of patients underwent laparoscopic surgery, while $30 \%(n=30)$ underwent laparotomy. The patients diagnosed with colon or rectal cancer were classified as stage I (20.86\%), stage II A (43.69\%), stage II b (6.50\%), stage III A (4.34\%), stage III B (17.01\%), and stage III C (7.60\%) following the TNM V classification.

The incidence of POD after elective FTS was 12.3\%. Delirium subtypes, according to DSMM, were classified as: hyperactive delirium $75 \%$; hypoactive delirium $20 \%$; mixed type $5 \%$. 
Table 4. Comparisons between predisposing factors, precipitating factors in delirious patients and nondelirious patients

\begin{tabular}{|c|c|c|c|}
\hline Clinical parameters & $\begin{array}{l}\text { Delirious } \\
(n=12)^{\mathrm{a}}\end{array}$ & $\begin{array}{l}\text { Nondelirious } \\
(n=85)^{\mathrm{a}}\end{array}$ & $p$ value $^{\mathrm{b}}$ \\
\hline Age, years & $80.02 \pm 0.45$ & $80.05 \pm 0.34$ & 0.5 \\
\hline 4AT score & $8.61 \pm 0.71$ & $2.68 \pm 0.07$ & $<0.0001$ \\
\hline ASA score & $2.28 \pm 0.43$ & $2.98 \pm 0.23$ & 0.5 \\
\hline MMSE score & $24.31 \pm 1.14$ & $27.56 \pm 0.34$ & $<0.02$ \\
\hline CIRS & $5.46 \pm 0.44$ & $4.22 \pm 0.20$ & 0.12 \\
\hline CDT & $3.36 \pm 0.38$ & $2.45 \pm 0.16$ & 0.09 \\
\hline MNA & $22.38 \pm 0.89$ & $23.47 \pm 0.16$ & 0.46 \\
\hline Barthel index & $93.08 \pm 2.56$ & $98.39 \pm 5.71$ & $<0.02$ \\
\hline IADL & $6.00 \pm 0.63$ & $7.37 \pm 0.15$ & 0.03 \\
\hline GDS & $3.84 \pm 1.03$ & $3.58 \pm 0.33$ & 0.95 \\
\hline Gijon scale & $10.08 \pm 0.81$ & $8.50 \pm 0.26$ & 0.33 \\
\hline Tinetti score & $19.69 \pm 1.94$ & $25.02 \pm 0.55$ & $<0.01$ \\
\hline NRS & $1.07 \pm 0.47$ & $0.53 \pm 0.19$ & $<0.04$ \\
\hline TUG score & $18.15 \pm 2.55$ & $9.63 \pm 0.42$ & $<0.02$ \\
\hline CGA score & $5.69 \pm 2.05$ & $3.14 \pm 0.24$ & $<0.005$ \\
\hline RI & $0.29 \pm 0.04$ & $0.22 \pm 0.01$ & 0.15 \\
\hline Dindo-Clavien score & $1.07 \pm 0.34$ & $1.07 \pm 1.19$ & 0.58 \\
\hline SF36 score & $0.90 \pm 0.26$ & $0.70 \pm 0.04$ & 0.43 \\
\hline Karnofsky score & $82.31 \pm 4.55$ & $90.48 \pm 1.16$ & 0.25 \\
\hline ECOG PS & $0.69 \pm 0.23$ & $0.32 \pm 0.06$ & 0.42 \\
\hline Hemoglobin, g/dL & $10.72 \pm 0.44$ & $10.90 \pm 0.16$ & 0.79 \\
\hline Sodium, MEq/L & $140.30 \pm 0.67$ & $140.7 \pm 0.33$ & 0.49 \\
\hline Potassium, MEq/L & $4.07 \pm 4.59$ & $4.06 \pm 0.05$ & 0.07 \\
\hline Creatinine, $\mathrm{mg} / \mathrm{dL}$ & $1.35 \pm 0.13$ & $1.16 \pm 0.04$ & 0.17 \\
\hline Mean drugs & $4.35 \pm 1.23$ & $4.01 \pm 0.11$ & 0.33 \\
\hline
\end{tabular}

MMMSE, Mini-Mental State Examination - cognitive status; CDT, Clock drawing test Shulman 1 visuospatial impairment; 4AT, rapid assessment test for delirium - screening test for delirium; CIRCS, Cumulative Illness Rate Scale for Geriatrics - multimorbidity; MNA, Mini-Nutritional Assessment - nutritional status; Barthel Index, functional status; IADL, Instrumental Activities of Daily Living - functional status; GDS, Geriatric Depression Scale - depression; Gijon scale - social frailty; Tinetti Scale - risk of falls; NRS, Numeric Rate Scale - pain; CGA, comprehensive geriatric assessment; RI, Rockwood 40-Item Index - frailty; DindoClavien score - postsurgical complications; ECOG Performance Status (ECOG score) - physical performance in oncology; Karnofsky score, physical performance in oncology; ASA, anesthesiologists' physical status; SF-36, 36-item Short Form Survey - quality of life; TUG, Timed up \& go. ${ }^{a}$ No missing data. ${ }^{\mathrm{b}}$ Parametric $t$ test.

The rate of postsurgical complications based on Dindo-Clavien assessment was $26 \%$ (grade 2: $11 \%$; grade 3: $3 \%$; grade 4: $4 \%$; and grade 5: 3\%). The rate of non-surgery-related adverse events was 3\% (urinary infection and upper respiratory disease), while $0.04 \%$ $(5 / 107)$ of patients needed postoperative blood transfusion. The mean length of in-hospital stay was $8.8 \pm 1.24$ days. No perioperative mortality ( 7 days after surgery) was recorded, while the 30 -day mortality rate was $4.95 \%$. Ninety percent of patients were discharged home, $5 \%$ of patients were admitted to intermediate care unit, while $5 \%$ of patients entered nursing homes for extensive physical rehabilitation.

Patients who developed postoperative delirium showed different clinical variables compared to nondelirious patients (Table 4). Namely, delirious patients were those more cognitively impaired, with decreased physical performance, increased functional decline, and reduced postural stability; these same patients showed a more significant impairment on the CGA. 
Furthermore, the multivariate analysis indicated that physical performance (TUG in seconds) was the most significant predictor of POD with an OR of 1.18 (95\% CI: 1.05-1.31; $p=0.005$ ) for each second of increase. Cognitive status (MMSE score) showed a trend in predicting POD (OR $=0.85$; 95\% CI: $0.71-1.01 ; p=0.068)$.

Patients submitted to laparoscopic procedure showed a lower trend in experiencing delirium, although the difference was not significantly different, compared to patients submitted to laparotomy ( $p=\mathrm{ns}$ ).

\section{Discussion}

FTS procedures in highly vulnerable oncogeriatric populations have not yet answered how effective they are in predicting the main clinical outcomes. To the best of our knowledge, this is the first study to investigate the risk factors for POD in a vulnerable octogenarian oncogeriatric population submitted to FTS surgery and frailty stratification.

In our study, the incidence of POD was $12.3 \%$, which is higher compared to the other procedure-specific studies. So far, few studies have examined the impact of FTS for colorectal cancer on POD. Namely, Krenk et al. [31] have shown no cases of POD after fast-track knee replacement surgery, compared to the usual incidence of $4-10 \%$.

Moreover, the fast-track setup in colonic oncogeriatric surgery was correlated with a shorter length of hospital stay and reduced incidence of POD (2.8\%) [32]. Recently, a subanalysis of the randomized clinical trial of Jia et al. [13] on patients over 80 years has indicated the protective role of FTS on postoperative complications, including delirium, in both elderly and oldest old patients [33]. In relation to these other studies, the heterogeneity associated with elderly populations and delirium assessment methodology may account for the wide range of these reported series. In particular, the findings of Kurbegovic et al. [32] were retrospective in nature, and there was no systematic geriatric assessment of patients' clinical vulnerability. Similarly, the study of Jia et al. [13], despite the lower incidence of POD (3.4\%) in patients submitted to FTS, did not include focused geriatric assessment of frailty.

Our relatively higher incidence of POD may be explained by the pre-frail phenotype of patients, which accounts for increased clinical vulnerability and decreased brain resilience.

It is noteworthy that the physical performance (TUG) was the best predictor of POD incidence, suggesting that the initial trajectory of frailty may be mostly linked to walking speed and physical ability [34]. Above-average physical performance probably reflects decreased resilience that characterizes these pre-frail categories of patients and predicts their decreased homeostasis and brain resilience in the presence of surgical stressors. These last features reflect the loss of structural and functional integrity and have been recently added to the concept of frailty [35]. In compliance with that, the current findings confirm frailty continuum as a key determinant predictor of POD [34] in surgical oncogeriatric patients as well.

Interestingly, the postoperative complication rate was $26 \%$, lower than the rates reported in the other procedure-specific studies $[13,32,33]$. The present findings confirmed the effectiveness of FTS in accelerating patient recovery and home discharge, even in vulnerable oncogeriatric patients.

The main limitations of this study were that it was carried out at a single institution and was relatively small in size. Delirium assessment was carried out at a single assessment point; thus, it did not include delirium duration, severity, or any change in clinical subtype as would be the case in longitudinal assessment. However, the single point assessment was established on the basis of the reference study by Jia et al. [13]. In compliance with that, the higher incidence of POD occurred at day 1 after FTS surgery, reflecting higher stressors such as anes- 
thetics, type of surgery, inflammatory response, and pain. Conversely, POD incidence during the course of hospital stay could be related to surgical and nonsurgical complications.

Notwithstanding these limitations and even if exploratory in nature, the study originally investigated the risk factors associated with POD, in major oncogeriatric colon surgery, after combining a clinical approach (FTS and CGA). Furthermore, the strength of the study lies in the accurate oncogeriatric assessment of "real world" patients' clinical vulnerability (pre-frail patients). Given the extent of the problem in the elderly vulnerable population undergoing cancer surgery, POD is a research top priority.

Future directions justify investigation of the development of FTS procedures integrated with effective frailty instruments to minimize harm after oncological surgery. Understanding the decreased inflammatory response after FTS in vulnerable oncogeriatric populations and their distinguished clinical trajectories of frailty may be of additional help in counteracting the devastating effect of this postoperative geriatric syndrome.

\section{Acknowledgments}

The authors are grateful to Prof. Alberto Ballestrero (DIMI, University of Genoa, Italy) and Dr. Roberto Murialdo (DIMI, University of Genoa, Italy) for data acquisition.

\section{Disclosure Statement}

No conflict of interest to disclose.

\section{Funding Sources}

No funding source to declare.

\section{Author Contributions}

Dr. Monacelli is responsible for study design and conception, drafting the manuscript, and critically revising the manuscript. Dr. Prefumo and Dr. Giannotti did data collection and the analysis and interpretation of data. Dr. Scabini and Dr. Romairone did data acquisition and performed the analysis and interpretation of data. Dr. Signori, Prof. Nencioni, and Prof Odetti revised the literature, did manuscript interpretation and critically revised the manuscript. All authors have read the paper, have agreed to be listed as authors and gave the final approval of the manuscript.

\section{References}

-1 Inouye SK, Westendorp RG, Saczynski JS, Kimchi EY, Cleinman AA: Delirium in elderly people - authors' reply. Lancet 2014;383:9934-2045.

12 Witlox J, Eurelings LS, de Jonghe JF, Kalisvaart KJ, Eikelenboom P, van Gool WA: Delirium in elderly patients and the risk of postdischarge mortality, institutionalization, and dementia: a meta-analysis. JAMA 2010;304: 443-451.

3 Minagawa H, Uchitomi Y, Yamawaki S, Ishitani K: Psychiatric morbidity in terminally ill cancer patients. A prospective study. Cancer 1996;78:1131-1137.

-4 Lawlor PG, Gagnon B, Mancini IL: Occurrence, causes, and outcome of delirium in patients with advanced cancer: a prospective study. Arch Int Med 2000;160:786-794. 
5 Takeuchi M, Takeuchi H, Fujisawa D: Incidence and risk factors of postoperative delirium in patients with esophageal cancer. Ann Surg Oncol 2012;19:3963-3970.

6 Bohner H, Hummel TC, Habel U: Predicting delirium after vascular surgery: a model based on pre- and intraoperative data. Ann Surg 2003;238:149-156.

7 Tei M, Wakasugi M, Kishi K, Tanemura M, Akamatsu H: Incidence and risk factors of postoperative delirium in elderly patients who underwent laparoscopic surgery for colorectal cancer. Int J Colorectal Dis 2016;31: 67-73.

-8 Kehlet H: Fast-track surgery - an update on physiological care principles to enhance recovery. Langenbecks Arch Surg 2011;396:585-590.

-9 Korc-Grodzicki B, Sun SW, Zhou Q, et al: Geriatric assessment as a predictor of delirium and other outcomes in elderly patients with cancer. Ann Surg 2015;261:1085-1090.

10 Dasgupta M, Dumbrell AC: Preoperative risk assessment for delirium after noncardiac surgery: a systematic review. J Am Geriatr Soc 2006;54:1578-1589.

$\checkmark 11$ Korc-Grodzicki B, Root JC, Alici Y: Prevention of post-operative delirium in older patients with cancer undergoing surgery. J Geriatr Oncol 2015;6:60-69.

12 Raats JW, Steunenberg SL, Crolla RM, Wijsman JH, te Slaa A, van der Laan L: Postoperative delirium in elderly after elective and acute colorectal surgery: a prospective cohort study. Int J Surg 2015;18:216-219.

13 Jia Y, Jin G, Guo S: Fast-track surgery decreases the incidence of postoperative delirium and other complications in elderly patients with colorectal carcinoma. Langenbecks Arch Surg 2014;399:77-84.

14 Rubenstein LZ, Josephson KR, Wieland GD, English PA, Sayre JA, Kane RL: Effectiveness of a geriatric evaluation unit. A randomized clinical trial. N Engl J Med 1984;311:1664-1670.

15 Folstein MF, Folstein SE, McHugh PR: "Mini-mental state": a practical method for grading the cognitive state of patients for the clinician. J Psychiatr Res 1975;12:189-198.

16 Shulman KI, Gold DP, Cohen CA, Zucchero CA: Clock-drawing and dementia in the community: a longitudinal study. Int J Geriatr Psychiatry 1993;8:487-496.

17 Mahoney FI, Barthel DW: Functional evaluation: the Barthel Index. Maryland State Med J 1965;14:61-65.

18 Lawton MP, Brody EM: Assessment of older people: self-maintaining and instrumental activities of daily living. Gerontologist 1969;9:179-186.

19 Conwell Y, Forbes NT, Cox C, Caine ED: Validation of a measure of physical illness burden at autopsy: the Cumulative Illness Rating Scale. J Am Geriatr Soc 1993;41:38-41.

20 Lesher EL, Berryhill JS: Validation of the Geriatric Depression Scale-Short Form among inpatients. J Clin Psychol 1994;50:256-260.

-21 Kaiser MJ, Bauer JM, Ramsch C: Validation of the Mini Nutritional Assessment short-form (MNA-SF): a practical tool for identification of nutritional status. J Nutr 2009;13:782-788.

22 Tinetti ME, Williams TF, Mayewski R: Fall risk index for elderly patients based on number of chronic disabilities. Am J Med 1986;80:429-434.

23 Rockwood K, Stadnyk K, MacKnight C, McDowell I, Hebert R, Hogan DB: A brief clinical instrument to classify frailty in elderly people. Lancet 1999;353:205-206.

24 Oken MM, Creech RH, Tormey DC: Toxicity and response criteria of the Eastern Cooperative Oncology Group. Am J Clin Oncol 1982;5:649-655.

25 New classification of physical status. Anesthesiology 1963;24:111.

-26 Podsiadlo D, Richardson S: The timed "Up \& Go": a test of basic functional mobility for frail elderly persons. J Am Geriatr Soc 1991;39:142-148.

27 European Delirium Association and American Delirium association: The DMS-5 criteria, level of arousal and delirium diagosis. BMC Med 2014;12:141.

28 Bellelli G, Morandi A, Davis DH: Validation of the 4AT, a new instrument for rapid delirium screening: a study in 234 hospitalised older people. Age Ageing 2014;43:496-502.

-29 Fitzgerald J, O’Regan N, Adamis D: Concordance between the delirium motor subtyping scale (DMSS) and the abbreviated version (DMSS-4) over longitudinal assessment in elderly medical inpatients. Intern Psychogeriatr 2016;28:845-851.

-30 Clavien PA, Barkun J, de Oliveira ML: The Clavien-Dindo classification of surgical complications: five-year experience. Ann Surg 2009;250:187-196.

-31 Krenk L, Rasmussen LS, Hansen TB, Bogo S, Soballe K, Kehlet H: Delirium after fast-track hip and knee arthroplasty. Br J Anaesth 2012;108:607-611.

-32 Kurbegovic S, Andersen J, Krenk L, Kehlet H: Delirium in fast-track colonic surgery. Langenbecks Arch Sur 2015;400:513-516.

-33 Day A, Fawcett WJ, Scott MJ, Rockall TA: Fast-track surgery and the elderly. Br J Anaesth 2012;109:124; author reply 124 .

-34 Abellan van Kan G, Rolland Y, Andrieu S: Gait speed at usual pace as a predictor of adverse outcomes in community-dwelling older people an International Academy on Nutrition and Aging (IANA) Task Force. J Nutr 2009;13:881-889.

-35 Lin HS, Peel NM, Hubbard RE: Baseline vulnerability and inpatient frailty status in relation to adverse outcomes in a surgical cohort. J Frailty Aging 2016;5:180-182. 